\title{
Selective attention in vibrotactile tasks: Detecting the presence and absence of amplitude change
}

\author{
KENNETH C. WHANG, HAROLD BURTON, and GORDON L. SHULMAN \\ Washington University School of Medicine, St. Louis, Missouri
}

\begin{abstract}
Selective spatial attention has a greater effect on detection of the absence of an amplitude change than it has on detection of the presence of such a change. Attention to one of four fingertips was manipulated by an $80 \%$ valid tactile cue in two-interval forced-choice tasks. In one task, the target was a vibrotactile amplitude change appearing among constant-amplitude distractors; in the other task, targets of constant amplitude had to be detected amid amplitude changes at the other fingertips. Cuing had a greater effect on the latter task than it did on the former. This asymmetry is consistent with the presence-absence asymmetry found in visual search and does not depend on the difficulty of the two tasks. A statistical model shows that a pooled activity mechanism could account for these experimental results.
\end{abstract}

Numerous tactile stimuli are present in one's environment at any given time-there may be considerable pressure against the reader's feet and seat, for example-but most of these stimuli escape notice unless one's attention is directed toward them. This introspection suggests that selective attention affects tactile perception; however, most of the experimental studies of this issue have failed to demonstrate attentive effects.

Experiments in which percent correct and reaction time (RT) have been used as performance measures have shown little or no effect of spatial attention on the detection of the presence of tactile intensity change. Shiffrin, Craig, and Cohen (1973) compared percentages of correct responses on a forced-choice localization task under two conditions. In the simultaneous condition, a pulse of vibration could appear at any of three positions on the hands and forearm during a single observation interval. In the sequential condition, the pulse could appear at only one of the three positions in each of three successive intervals. The subjects could therefore direct their attention to each position in turn, instead of dividing it among all three positions simultaneously. Localization of the pulse of vibration was no better in the sequential condition than it was in the simultaneous condition, leading the authors to conclude that spatial attention does not affect tactile

\footnotetext{
The present research was supported by the following grants: ONR N-00014-86-0289, PHS 5 T32 NS07507-11, and NIDCD 00096. The authors thank Steven Petersen and Michael Posner for providing experimental facilities, Michael Posner and K. Sathian for criticizing the manuscript, Edward Spitznagel for advice on statistical analysis, Arnold Heidbreder and Janet Weisenberger for providing equipment and technical help, and all of the subjects for their participation. Correspondence should be addressed to Kenneth C. Whang, Box 8108, 660 South Euclid Avenue, Washington University School of Medicine, St. Louis, MO 63110.
}

perception. An analogous visual experiment yielded similar results (Shiffrin \& Gardner, 1972).

Posner (1978) examined the effect of a probability cue on tactile RTs. Asked to respond as quickly as possible to a tap on the left or right index fingertip, subjects were cued at the start of each trial by an arrow pointing to the likely position of the tap. The arrow correctly predicted target position on $80 \%$ of the trials; these trials were said to be validly cued. If detection of the tap demanded spatial attention, the subjects were expected to attend to the cued position and, consequently, to respond more quickly on validly cued trials than on invalidly cued trials. If the task did not demand spatial attention, no RT difference was expected. The effect of cuing was not significant in this experiment, in contrast to the analogous visual experiment, which demonstrated a significant RT difference for detection of a luminance change. In a similar RT study, using lateralized visual and tactile stimuli as probability cues, Butter, Buchtel, and Santucci (1989) found that the effect of cuing on tactile detection was significant, but smaller than the corresponding effect on visual detection. Thus, it appears that spatial attention has a small effect at best on simple tactile detection tasks.

Posner (1978) found a larger effect of cuing on a tactile $\mathrm{RT}$ task in which subjects responded only to the weaker of two possible stimuli. Unlike the detection task, this task required subjects to perform a tactile discrimination to avoid responding to energy alone. Posner suggested that energy detection occurred preattentively in the tactile modality, but that discrimination demands attention.

Visual search experiments have shown that for a variety of visual features, detection of the absence of the feature demands greater spatial attention than does detection of its presence (Treisman \& Gormican, 1988; Treisman $\&$ Souther, 1985). Search for a target distinguished from distractors by the presence or greater intensity of a fea- 


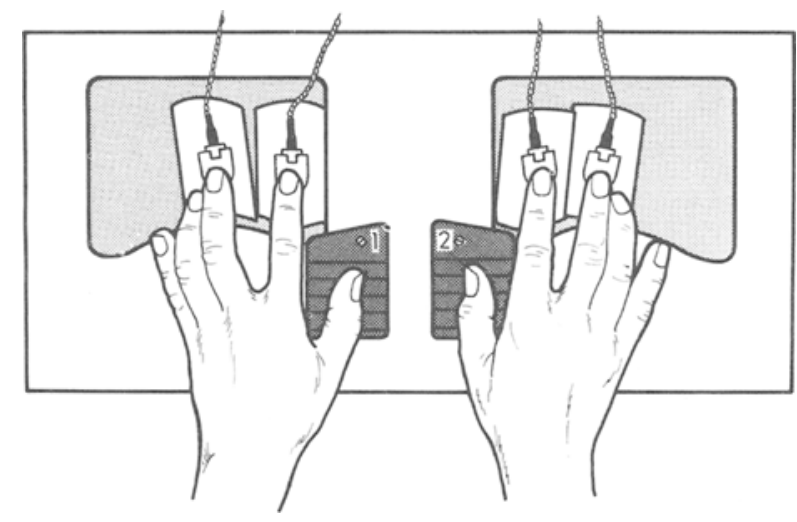

Figure 1. Finger stimulators.

ture, such as line length or luminance contrast, was nearly as rapid when the target appeared in an array among several distractors as when it appeared alone in the display. When the target and distractor were switched, so that the target was distinguished from distractors by feature absence or less intensity, search time increased linearly with the number of items in the stimulus array, consistent with the hypothesis that the display was scanned in a serial, rate-limited fashion.

If Treisman's findings generalize to the tactile modality, a similar asymmetry may be expected between detection of the presence and absence of tactile features. This is the issue that the present experiments were designed to address.

\section{EXPERIMENT 1}

An $80 \%$ valid probability cue was used to manipulate attention to the fingertips in two tasks, hereafter referred to as the presence and absence tasks. In the presence task, subjects tried to detect the presence of a vibrotactile amplitude change over a constant-amplitude base vibration. In the absence task, the target was the base vibration with no amplitude change. Performance was measured in terms of percent correct on two-interval forced-choice trials. Stimulus amplitudes were varied so that performance levels on the two tasks fell in the same range, and differences between the presence and absence tasks could be distinguished from more general effects of task difficulty.

\section{Method}

Subjects. Three men and four women, 22-29 years old, were paid $\$ 10$ per session for five or six sessions, each lasting 1-2 $\mathrm{h}$. All were students or technicians at Washington University. Three had participated in previous tactile experiments in the laboratory. All 7 wrote with their right hands.

Apparatus. The apparatus shown in Figure 1 delivered vibratory stimuli to the subjects' index and middle fingertips. The subjects rested their hands on a wooden board as shown, with their thumbs on response keys and fingertips over a pad of foam batting. Positioned atop the batting under each stimulated fingertip was a skin vibrator, fixed in place by Velcro.

The vibrators were designed by David Franklin of Audiological Engineering (Somerville, MA; Model V1220). Each consisted of a coil and mass suspended over a magnet by a $U$-shaped leaf spring. The magnetic field acted on current in the coil to generate a force proportional to the applied input voltage, causing the assembly and attached plastic enclosure to vibrate. When operated near the resonance frequency of $260 \mathrm{~Hz}$, the devices vibrated well above

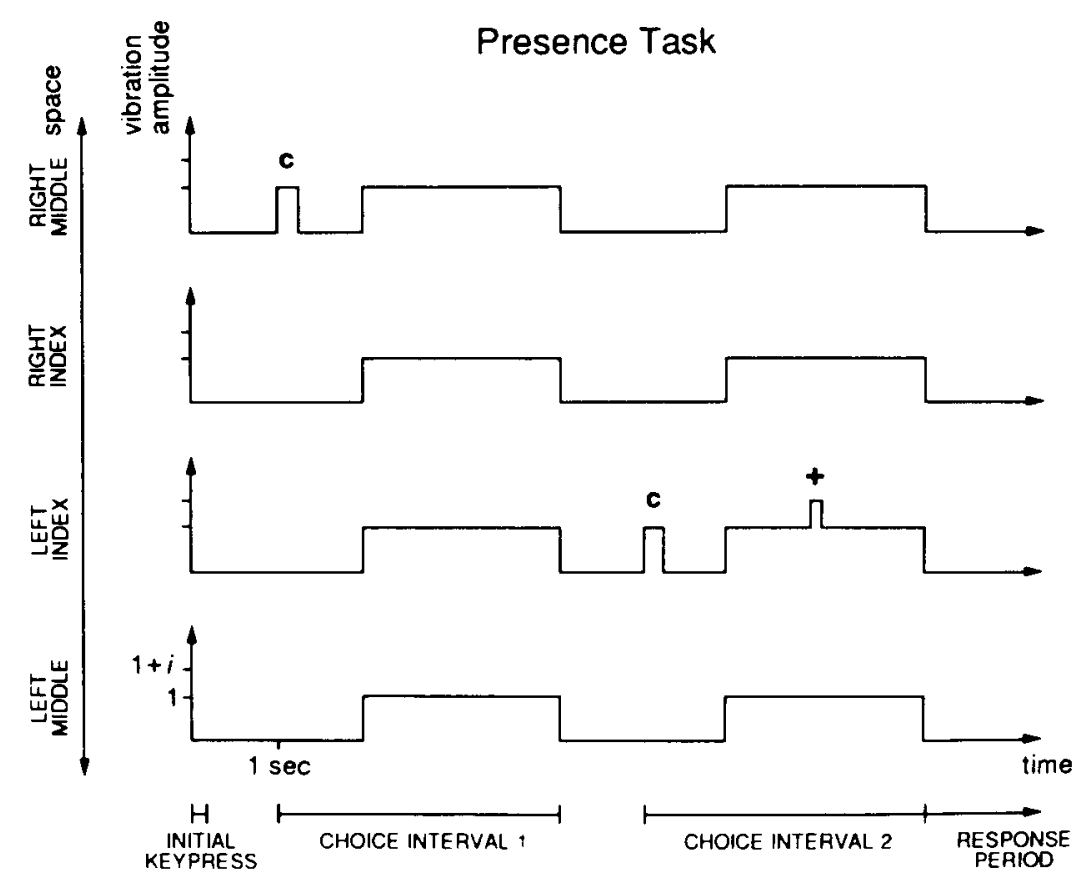

Figure 2. Presence task. Amplitudes of vibration presented to each fingertip are plotted as functions of time. The target, a 50 -msec pulse of increased amplitude, is marked with $a$ ". "Tactile spatial cues for each choice interval are marked with the letter "c." 
tactile threshold while making only a faint humming sound. Contact area between the plastic case and the skin was limited by curvature of the fingertip to about $1 \mathrm{~cm}^{2}$.

Variable-amplitude, $250-\mathrm{Hz}$ sinusoids drove the stimulators. The direct output from a function generator as well as an attenuated, in-phase copy of the waveform were switched on and off, summed, and amplified by a switching circuit controlled by an Apple Ile computer. Signals to each stimulator were thereby stepped independently to any of four discrete levels. The frequency and amplitude of the inputs to the switching circuit were monitored by a frequency counter and a voltmeter.

Procedure. Presented with a target and three distractors in one choice interval, and with distractors at all four positions in the other, the subjects were asked to determine in which choice interval the target occurred. Note that the task was to identify the choice interval, not the position of the target, since spatial cuing would bias the latter judgment.

In the presence task, the target was a 50-msec pulse of increased amplitude over a base vibration. The pulse began $1 \mathrm{sec}$ after the onset of the base vibration, which lasted for $2.3 \mathrm{sec}$. The distractor was the base vibration presented with no change in amplitude. In the example diagrammed in Figure 2, the amplitude change occurs at the left index fingertip in the second choice interval, so the correct response for the trial would be " 2 ."

A tactile spatial cue preceded the stimuli in each choice interval. At one of the four fingertips, a 250 -msec pulse of vibration appeared $1 \mathrm{sec}$ before onset of the base vibration to indicate the likely position of the target. In $80 \%$ of the trials, the target was presented to the same fingertip as was the cue, as in the example in Figure 2. The cue in the target-absent interval was random, appearing at any of the four fingertips with equal probability.

The absence task was identical to the presence task, except that the target and distractor were switched. The subjects determined in which choice interval constant-amplitude vibration was presented to one of the fingertips, as pulses of increased amplitude were presented elsewhere. In the example diagrammed in Figure 3, there is no amplitude change at the left middle fingertip in the first choice interval, so the correct response would be " 1 ." Cuing in this example is invalid.

The subjects responded by pressing the left key, labeled " 1 ,", for the first choice interval, or the right key, labeled " 2 ," for the second. Most of the subjects responded within 200-1,500 msec following the end of the stimuli; they were allowed as much time as they wanted. Immediate feedback was given for each response: two short, high-pitched tones from the computer speaker for a correct response, or a longer, low-pitched tone for an incorrect response. After the feedback period, either key could be pressed to initiate the next trial. Performance was measured in terms of percentage of correct responses under each task condition in blocks of 120 trials presented in randomized order.

Each subject ran in two or three blocks of the presence task and three blocks of the absence task. Different blocks were presented on separate days in randomized order. The sizes of the amplitude changes were adjusted to make the task easier on some blocks and more difficult on others. The base amplitude was fixed at approximately $6.7 \mathrm{~V}$ rms for all subjects.

For the presence task, the amplitude changes were set so that overall performance levels on easy and difficult blocks were above and below $70 \%$ correct, respectively, for all subjects. The amplitude changes used for each subject are listed in Table 1 . Two subjects ran in three blocks instead of two because their performance levels on the first two blocks were both above or both below $70 \%$ correct. Data from the extra blocks were treated as within-subjects replicates.

For the absence task, it was more difficult to standardize amplitude in terms of performance. Some subjects performed progressively better as the size of the amplitude change increased. Others found that past a point, further increases in distractor size made the task more difficult. The amplitude changes were crudely grouped into the small, medium, and large ranges listed in Table 1 . All subjects required larger amplitude changes on the absence task than they did on the presence task to perform at the same level.

The subjects did at least 30 practice trials at the beginning of each session; they did more if necessary, in order for them to feel comfortable with the task. On the 1st day, they typically began with

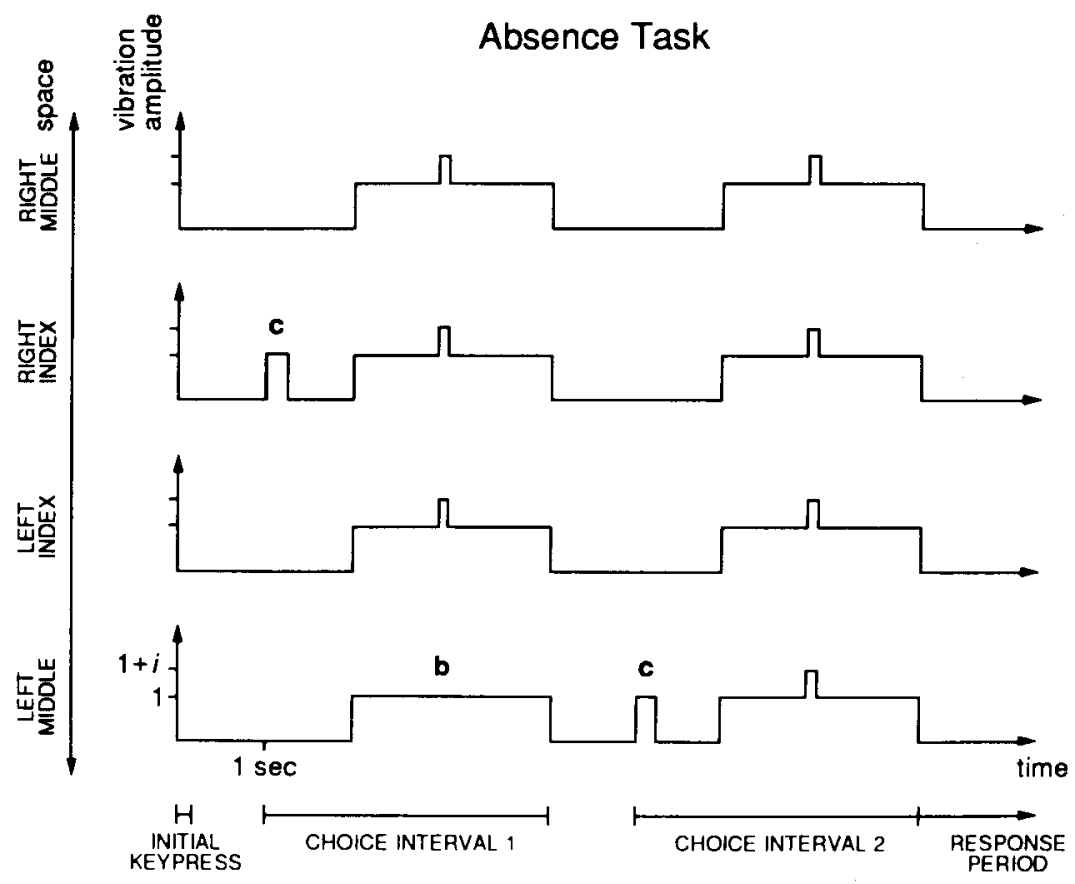

Figure 3. Absence task. The target, a constant-amplitude vibration, is marked with a "b." 
Table 1

Experiment 1 Amplitude Changes

\begin{tabular}{lcccccc}
\hline & \multicolumn{2}{c}{ Presence Task } & & \multicolumn{3}{c}{ Absence Task } \\
\cline { 2 - 3 } \cline { 5 - 7 } Subject & Small & Large & & Small & Medium & Large \\
\hline B.S. & .23 & .26 & & .31 & .80 & 1.45 \\
F.J. & .57 & .80 & & .89 & 1.00 & 1.12 \\
J.L. & .17 & .26 & & .31 & .89 & 1.45 \\
K.S. & .26 & .39 & & .57 & .80 & 1.45 \\
S.K. & .26 .31 & .39 & & .69 & 1.00 & 1.45 \\
S.S. & .26 & .31 & & .39 & .89 & 1.45 \\
Z.X. & .26 & .39 .57 & & .47 & 1.00 & 1.25 \\
Mean $i$ & .29 & .42 & & .52 & .91 & 1.37 \\
\hline
\end{tabular}

Note-Sizes of the amplitude changes, $i$, are expressed as fractions of the base vibration amplitude, measured in units of rms voltage to the stimulators.

60-90 practice trials. During these practice trials, the amplitude changes were adjusted to the appropriate difficulty.

The subjects took breaks to rest for a few minutes after every 30 trials.

\section{Results and Discussion}

Percentages of correct responses were analyzed by repeated measures analysis of variance. Spatial cuing was treated as a factor with four levels: valid, ipsilateral to target, contralateral on the homologous fingertip, and contralateral on the nonhomologous fingertip. (For a target on the right middle fingertip, these levels would correspond to cuing to the right middle, right index, left middle, and left index fingertips, respectively.) Analyses of a variance stabilizing transform of percent correct $(\theta=$ $\arcsin \sqrt{p}$; Fisher, cited in Box, Hunter, \& Hunter, 1978) yielded similar results.

Analysis of the combined data from both tasks showed a significant overall effect of cuing $[F(3,18)=7.80$, $p<.002]$ and a significant interaction between task and cuing $[F(3,18)=4.40, p<.02]$. Separate analyses of the data from each task showed that the effect of cuing was not significant in the presence task $[F(3,18)=1.51]$, but that it was significant in the absence task $[F(3,18)=$ $11.13, p<.0005$ ]. Post hoc paired comparisons of the absence task data indicated that performance on validly cued trials was significantly higher than performance under any of the three invalid cuing conditions (Scheffé's test, $p<.05$ ), but none of the invalid cuing conditions differed significantly from another. Performance means are summarized in Table 2.
Reducing the size of the amplitude change lowered performance on the presence task $[F(1,6)=13.05, p<.02]$, but it did not interact with the cuing manipulation $[F(3,18)$ $=1.05]$. In the absence task, cuing and size of the amplitude change did interact $[F(6,36)=4.39, p<.005]$, but this resulted from a "floor" effect: As the size of the distractor amplitude changes increased, performance on validly cued trials increased while performance on invalidly cued trials stayed at chance.

None of the other factors tested-target hand (left or right), target digit (index or middle), or target choice interval-affected performance significantly on either task.

The results of Experiment 1 suggest that tactile spatial attention affects the absence task more than it affects the presence task; however, it is possible that auditory cues could have contributed to this asymmetry. Since the vibrators were faintly audible, the subjects may have detected a slight difference in sound between the target-present and target-absent intervals in the presence task. Such a difference would have been much harder to detect in the absence task, since multiple amplitude changes were presented in both intervals.

\section{EXPERIMENT 2}

To control for the possible effects of auditory cues, a second group of subjects was tested on the presence and absence tasks, with the sound of the vibrators masked by pink noise.

\section{Method}

Subjects. Five women and 5 men, 22-29 years old, served as the subjects in Experiment 2. All were right-handed students or technicians at Washington University. Two had participated in previous tactile experiments; none in Experiment 1.

Apparatus. Vibrotactile stimuli were presented with the same apparatus as was used in Experiment 1. Pink noise (General Radio $1390 \mathrm{~B}$ random-noise generator; $1390-\mathrm{P} 2$ pink noise filter) was presented over headphones at $70 \mathrm{~dB}$ SPL.

Procedure. The two tasks were the same as in Experiment 1. The only difference in procedure was that at the end of each trial, the experimenter said "yes" or "no" to indicate correctness of the response. Most subjects had difficulty hearing the computer tones over the pink noise, but all reported that they could hear the experimenter's voice clearly.

Each subject ran in one block each of the presence and absence tasks. The order of blocks was counterbalanced across subjects. Amplitudes were chosen to keep overall performance at compara-

Table 2

Experiment 1 Performance Data

\begin{tabular}{lccccccc}
\hline & \multicolumn{2}{c}{ Presence } & Task & & \multicolumn{3}{c}{ Absence Task } \\
\cline { 2 - 3 } \cline { 7 - 8 } \multicolumn{1}{c}{ Cuing } & Small & Large & & Small & Medium & Large \\
\hline Valid & 63.7 & 82.4 & & 68.1 & 78.4 & 79.1 \\
Ipsilateral & 60.9 & 82.8 & & 67.9 & 53.6 & 50.0 \\
Contralateral-homologous & 54.7 & 70.3 & & 48.2 & 58.9 & 57.1 \\
Contralateral-nonhomologous & 62.5 & 73.4 & & 55.4 & 35.7 & 48.2 \\
\hline
\end{tabular}

Note-Mean percentages of correct responses in the presence and absence tasks are listed for each amplitude and cuing condition. 
Table 3

Experiment 2 Amplitude Changes

\begin{tabular}{lcc}
\hline Subject & $\begin{array}{c}\text { Presence } \\
\text { Task }\end{array}$ & $\begin{array}{c}\text { Absence } \\
\text { Task }\end{array}$ \\
\hline B.P. & .38 & 2.26 \\
C.V. & .30 & 0.90 \\
D.B. & .30 & 1.00 \\
D.S. & .27 & 1.28 \\
J.D. & .30 & 1.84 \\
L.T. & .27 & 2.26 \\
R.R. & .30 & 1.00 \\
S.C.S. & .24 & 1.84 \\
S.E. & .34 & 2.26 \\
S.T. & .30 & 3.50 \\
Mean $i$ & .30 & 1.81 \\
\hline
\end{tabular}

Table 4

Experiment 2 Performance Data

\begin{tabular}{lcc}
\hline Cuing & Presence Task & Absence Task \\
\hline Valid & 76.0 & 75.8 \\
Invalid & 70.8 & 50.0 \\
\hline
\end{tabular}

ble levels on the two tasks. The amplitude changes used for each subject are summarized in Table 3.

\section{Results and Discussion}

A repeated measures analysis of variance with cuing as a two-level factor (valid or invalid) demonstrated a significant overall effect of cuing on the two tasks $[F(1,9)$ $=13.74, p<.005]$ and a significant interaction between task and cuing $[F(1,9)=5.89, p<.05]$. As in Experiment 1 , separate analyses of the data from each task showed a significant effect of cuing on the absence task $[F(1,9)=33.51, p<.0005]$, but not the presence task $[F(1,9)=1.96]$. Means under each condition are summarized in Table 4.

When cuing was treated as a four-level factor (valid, ipsilateral, contralateral-homologous, contralateral-nonhomologous), the task $\times$ cuing interaction was not significant $[F(3,27)=1.48]$. This inconsistency with Experiment 1 may have been due to the smaller number of trials per subject in Experiment 2. In Experiment 1, the task $\times$ cuing interaction in the two-level analysis was marginal $[F(1,6)=5.35, p=.06]$. Significance tests of all other effects yielded the same results in the four-level analyses as in the two-level analyses of both experiments.

The results of Experiment 2 confirm the attentional asymmetry between the presence and absence tasks and show that it does not depend on auditory cues. In the context of Treisman's visual search experiments (Treisman \& Gormican, 1988; Treisman \& Souther, 1985), the asymmetry suggests that vibratory amplitude change may be a preattentively processed feature in the tactile modality. Similar asymmetries have been found in detection of the presence and absence of tactile texture changes (Sathian \& Burton, in press).

\section{GENERAL DISCUSSION}

Two main findings must be accounted for. First, the task $\times$ cuing interactions in both experiments show that the absence task demands greater attention than the presence task does. Second, the amplitude manipulation in Experiment 1 shows that this asymmetry does not depend on a difference in task difficulty.

The asymmetry of the present results cannot be accounted for by discriminability of targets and distractors at the level of the individual stimulus position. Duncan and Humphreys (1989) have proposed that limited resources are allocated most efficiently when distractors are dissimilar to targets and similar to each other. In both tasks of the present experiments, the discrimination at single fingertips was between the presence and absence of amplitude change. Target and distractor amplitudes were more dissimilar in the absence task than they were in the presence task, yet only the absence task showed a significant attentional effect.

We must therefore consider the context in which the targets and distractors were presented: in the presence task, among feature-absent stimuli (constant amplitude), or in the absence task, among feature-present stimuli (amplitude changes).

One possible framework for interpretation is suggested by Duncan (1980). Using a paradigm similar to that of Shiffrin and Gardner (1972), Duncan found that distinguishing visual displays with one target from those with none demanded less attention than did distinguishing displays with two targets from those with just one. He explained this by hypothesizing that distractors do not compete for limited-capacity attentive processes. It follows that there is no attentional interference in the former task, but that detection of one target interferes with simultaneous detection of a second target in the latter task. If instead of viewing the absence task as a discrimination between one feature-absent target and none, one considers it to be a discrimination between four feature-present targets and three, then the present results are consistent with Duncan's theory.

Another framework has been offered by Treisman and Souther (1985). They proposed that target-present and target-absent stimulus arrays may be distinguished on the basis of pooled feature-detector activities, and explained presence-absence asymmetry in terms of Weber's law. Suppose that the activities of feature detectors representing the entire stimulus array are pooled. The absolute difference between the summed activities evoked by targetpresent and target-absent arrays is the same in the presence task as it is in the absence task; both cases reduce to the difference between a feature-present stimulus and a featureabsent one. However, this difference occurs in the context of low background activity in the presence task, since distractors are defined by feature absence, and in the context of high background activity in the absence task. Ac- 
cording to Weber's law, the activity change is therefore harder to detect in the absence task unless one lowers background activity by narrowing the attentional focus over which activity is pooled.

Both of these interpretations account for the disparate results of the cuing manipulation at a qualitative level, without requiring separate mechanisms to govern the attentional demands of the two tasks. At a quantitative level, Treisman and Souther (1985) have also proposed an index of predicted performance against which their model can be evaluated. Their quantification in terms of Weber fractions, however, is not consistent with the results of the amplitude manipulation.

The amplitude manipulation in Experiment 1 shows that the attentional demands of the presence and absence tasks are not related to task difficulty. Between tasks, amplitudes were adjusted to keep overall performance in the same range, yet there were significant interactions between task and cuing. Within tasks, increasing difficulty did not bring out a cuing effect in the presence task, and decreasing difficulty did not bring invalid performance above chance in the absence task.

The Weber fraction model predicts, on the contrary, that task difficulty should affect attentional demand. In the presence task, attentional narrowing of the pooling area increases the ratio of activity change to background on validly cued trials. An attentive strategy should therefore improve overall performance unless nonattentive performance already reaches ceiling. If performance under easier amplitude conditions is at ceiling, then reducing the size of the amplitude change should bring performance down to a range within which attention has an effect. A simulation based on the Weber fraction model confirmed these predictions, showing that the model could not adequately account for the findings of Experiment 1 .

An alternative model is proposed, in which performance is statistically quantified in terms of means and variances of pooled feature-detector activities. In this model, it is assumed that the variances in total activity of populations of detectors increase with the mean activities, and further, that propagated variance at the level of a pooled activity unit limits one's ability to resolve the difference in means between target-present and target-absent stimulus arrays. Spatial attention is represented by the relative weighting of inputs to the pooled activity from detector populations representing different stimulus positions.

A simulation of this model, described more fully in the Appendix, showed that the predicted probabilities of correct responses are close to the human performance data (Table 5) under the following conditions:

1. The feature detectors are highly selective for transient amplitude change.

2. The minimum weight of inputs to the pooled activity unit is between $20 \%$ and $40 \%$ of the maximum.

3 . The activities of detector populations representing different fingertips are highly correlated.

The predicted cuing asymmetry depends most critically on Condition 1. Condition 2 affects the size of the cuing
Table 5

Model Predictions Compared to Performance Data

\begin{tabular}{|c|c|c|c|c|c|c|}
\hline \multirow[b]{2}{*}{ Task } & \multirow[b]{2}{*}{ Cuing } & \multicolumn{5}{|c|}{ Amplitude Change $i$} \\
\hline & & .29 & .42 & .52 & .91 & 1.37 \\
\hline \multicolumn{7}{|c|}{ Performance Data } \\
\hline $\begin{array}{l}\text { Presence } \\
\text { Presence }\end{array}$ & $\begin{array}{l}\text { valid } \\
\text { invalid }\end{array}$ & $\begin{array}{l}63.7 \\
59.4\end{array}$ & $\begin{array}{l}82.4 \\
75.5\end{array}$ & & & \\
\hline $\begin{array}{l}\text { Absence } \\
\text { Absence }\end{array}$ & $\begin{array}{l}\text { valid } \\
\text { invalid }\end{array}$ & & & $\begin{array}{l}68.1 \\
57.2\end{array}$ & $\begin{array}{l}78.4 \\
49.4\end{array}$ & $\begin{array}{l}79.1 \\
51.8\end{array}$ \\
\hline \multicolumn{7}{|c|}{ Model Predictions } \\
\hline $\begin{array}{l}\text { Presence } \\
\text { Presence }\end{array}$ & $\begin{array}{l}\text { valid } \\
\text { invalid }\end{array}$ & $\begin{array}{l}63.9 \\
63.9\end{array}$ & $\begin{array}{l}76.6 \\
76.6\end{array}$ & $\begin{array}{l}83.7 \\
83.7\end{array}$ & $\begin{array}{l}93.1 \\
93.1\end{array}$ & $\begin{array}{l}94.4 \\
94.4\end{array}$ \\
\hline $\begin{array}{l}\text { Absence } \\
\text { Absence }\end{array}$ & $\begin{array}{l}\text { valid } \\
\text { invalid }\end{array}$ & $\begin{array}{l}57.2 \\
52.0\end{array}$ & $\begin{array}{l}64.5 \\
54.0\end{array}$ & $\begin{array}{l}69.3 \\
55.4\end{array}$ & $\begin{array}{l}77.7 \\
58.2\end{array}$ & $\begin{array}{l}79.2 \\
58.7\end{array}$ \\
\hline
\end{tabular}

effect seen in the absence task. Condition 3 affects the relative overall difficulties of the two tasks.

The pooling mechanism assumed in the model is consistent with physiological effects that have been observed in the large visual receptive fields of V4 and IT neurons (Moran \& Desimone, 1985). Response to an optimal visual stimulus was found to be attenuated when attention was directed to another stimulus within the same receptive field. Interestingly, the degree of attenuation found in V4 (medians of $33 \%$ and $36 \%$ for two stimulus conditions) was in the same range as the analogous weight ratios predicted by the model.

Pooling of tactile responses across a broad spatial extent is suggested by psychophysical studies in which contact area has been manipulated. Verrillo (1963) found that increasing the contact area of a high-frequency stimulator decreased the threshold for detection. Craig (1968) found that an equivalent drop in threshold resulted when contact area was doubled by adding a second stimulus elsewhere on the body, far from the first stimulus. Physiological responses in SII and area $7 \mathrm{~b}$ are characterized by large receptive fields, many of them bilateral (Burton, 1986; Robinson \& Burton, 1980). Cells in these areas might be able to pool activities from lower cortical levels selectively and thereby contribute to the attentional effects that have been demonstrated in the present experiments.

\section{REFERENCES}

Box, G. E. P., Hunter, W. G., \& Hunter, J. S. (1978). Statistics for experimenters. New York: Wiley.

BurToN, H. (1986). Second somatosensory cortex and related areas. In E. G. Jones \& A. Peters (Eds.), Cerebral cortex (Vol. 5, pp. 31-98). New York: Plenum.

Butter, C. M., Buchtel, H. A., \& Santucci, R. (1989). Spatial attentional shifts: Further evidence for the role of polysensory mechanisms using visual and tactile stimuli. Neuropsychologia, 27, 1231-1240.

Craig, J. C. (1968). Vibrotactile spatial summation. Perception \& Psychophysics, 4, 351-354.

DUnCAN, J. (1980). The locus of interference in the perception of simultaneous stimuli. Psychological Review, 87, 272-300.

DunCAN, J., \& HumphreYs, G. W. (1989). Visual search and stimulus similarity. Psychological Review, 96, 433-458. 
Moran, J., \& Desimone, R. (1985). Selective attention gates visual processing in extrastriate cortex. Science, 229, 782-784.

Posner, M. I. (1978). Chronometric explorations of mind. Hillsdale, NJ: Erlbaum.

RoBinson, C. J., \& BURTON, H. (1980). The organization of somatosensory receptive fields in cortical areas $7 \mathrm{~b}$, retroinsular, postauditory and granular insula of $\mathrm{M}$. fascicularis. Joumal of Comparative Neurology, 192, 69-92.

Sathian, K., Burton, H. (in press). The role of spatially selective attention in the tactile perception of texture. Perception \& Psychophysics.

Shiffrin, R. M., Craig, J. C., \& Cohen, E. (1973). On the degree of attention and capacity limitation in tactile processing. Perception \& Psychophysics, 13, 328-336.

ShIfFrIn, R. M., \& GARDNer, G. T. (1972). Visual processing capacity and attentional control. Joumal of Experimental Psychology, 93, $72-82$.

Treisman, A., \& Gormican, S. (1988). Feature analysis in early vision: Evidence from search asymmetries. Psychological Review, 95 , 15-48.

Treisman, A., \&outher, J. (1985). Search asymmetry: A diagnostic for preattentive processing of separable features. Journal of Experimental Psychology: General, 114, 285-310.

VERRILL, R. T. (1963). Effect of contactor area on the vibrotactile threshold. Journal of the Acoustical Society of America, 35, 1962-1966.

\section{APPENDIX}

\section{Premises}

The premises of the model are illustrated in Figure Al. A pooled activity unit $(I)$ receives a weighted sum of activities $\left(a_{1}\right.$ through $a_{4}$ ) from detector populations representing the four stimulated fingertips. The relative weighting of each fingertip $\left(w_{1}\right.$ through $\left.w_{4}\right)$ corresponds to the allocation of spatial attention. Weights between $w_{\min }$ and $w_{\max }$ are chosen to maximize overall performance on validly and invalidly cued trials.

Activities of these populations of detectors are postulated to increase in both mean $\left(\mu_{c}\right)$ and variance $\left(\sigma_{a}^{2}\right)$ in response to vibratory amplizude change at the corresponding fingertips. Although any scheme involving concomitant increases in mean and variance would lead to qualitatively similar predictions, it is assumed specifically that these increases result from recruitment of additional detector units, whose logarithmic thresholds are distributed normally with parameters $\mu_{0}$ and $\sigma^{z}$. Each active detector $(u)$ contributes a mean $\left(\mu_{u}\right)$ and variance $\left(\sigma_{u}^{2}\right)$ to the population activity; inactive detectors $(0)$ are assumed to contribute nothing. The stimulus at a single fingertip is either the base vibration alone $(b)$ or the base plus a transient increase $(b+i)$. The
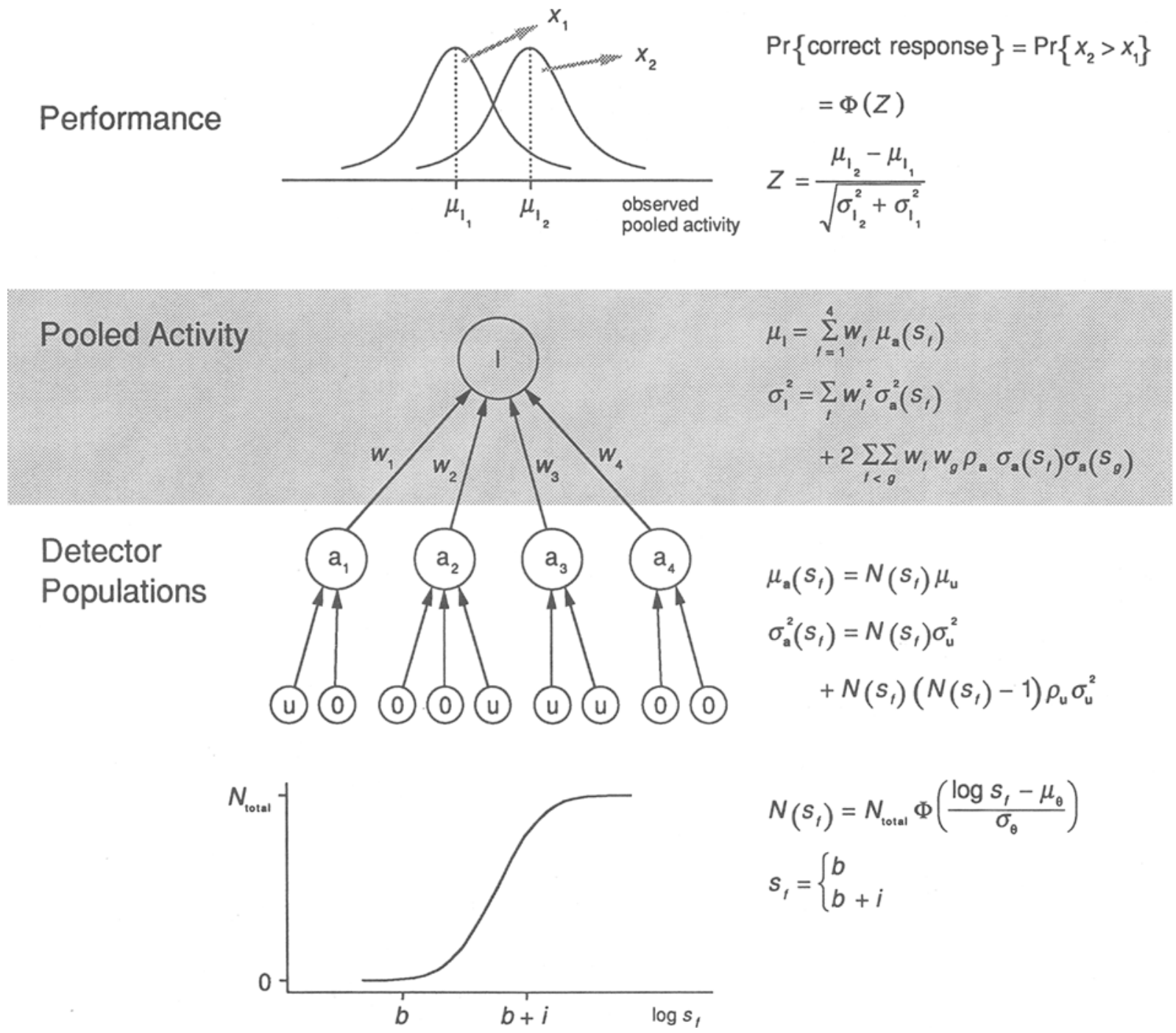

Figure A1. Premises of the model. See text for details. 

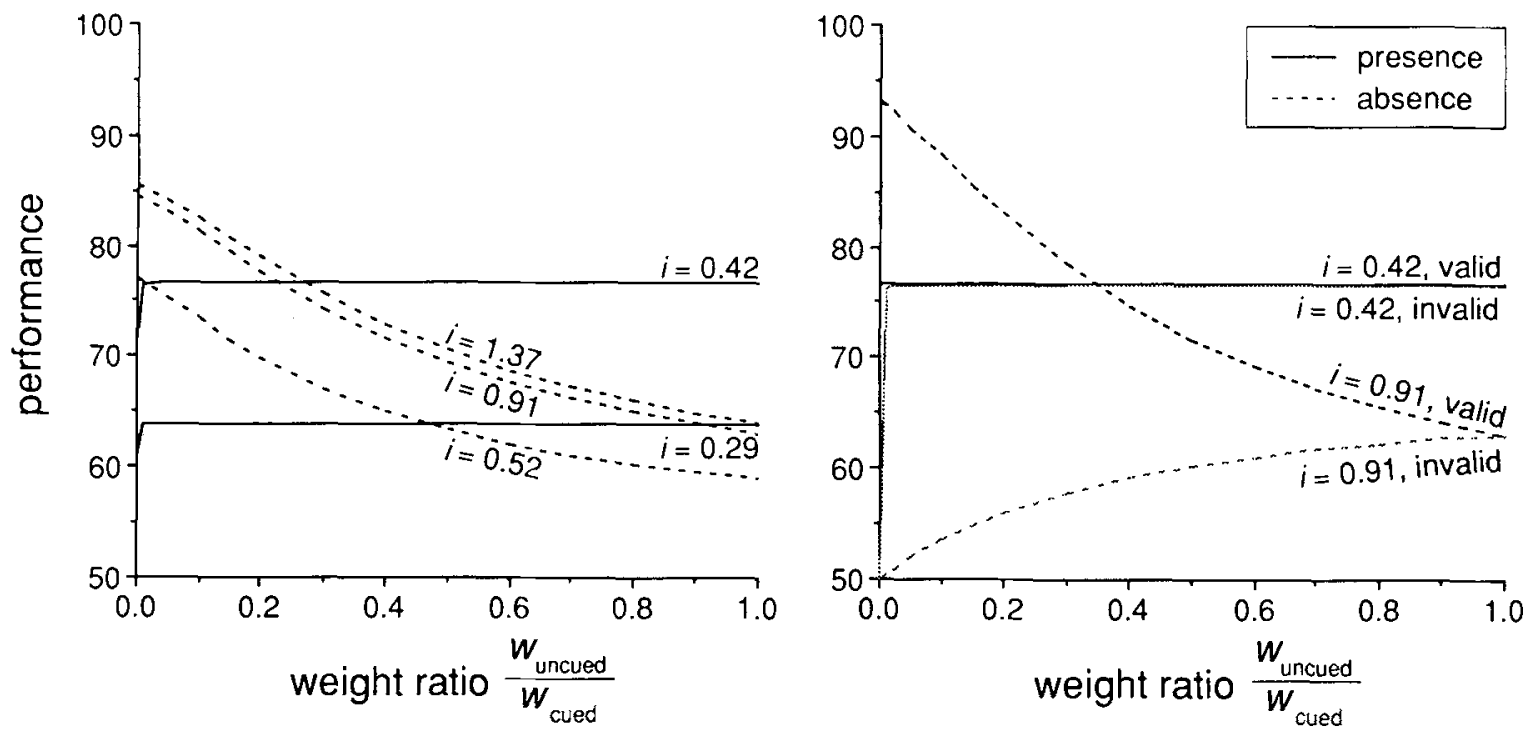

Figure A2. Left: Predicted overall performance on the presence (solid lines) and absence (dashed lines) tasks as a function of weight ratio $w_{\text {uncued }} / w_{\text {cued. }}$. Model parameters are the same as in Table $5: b=0.1, \rho_{a}=0.7, \mu_{u} / \sigma_{u}=1.6, \mu_{e}=-0.37, \sigma_{e}^{2}=0.12$. Right: Predicted performance at selected amplitudes under valid (black lines) and invalid (gray lines) cuing conditions.

values of $i$ are the averages across subjects listed in Tables 2 and 5 . The parameter $b$ represents the relative responsiveness to the steady-state base vibration compared to a transient vibration of the same amplitude. A lower value for $b$ represents greater habituation to steady-state vibration and greater selectivity for transients.

The component means and variances of the activities of detector populations are reflected in the mean $\left(\mu_{I}\right)$ and variance $\left(\sigma_{I}^{2}\right)$ of the pooled activity. The mean pooled activity is equal to the weighted sum of the mean population activities; the variance is equal to the square-weighted sum of the input variances

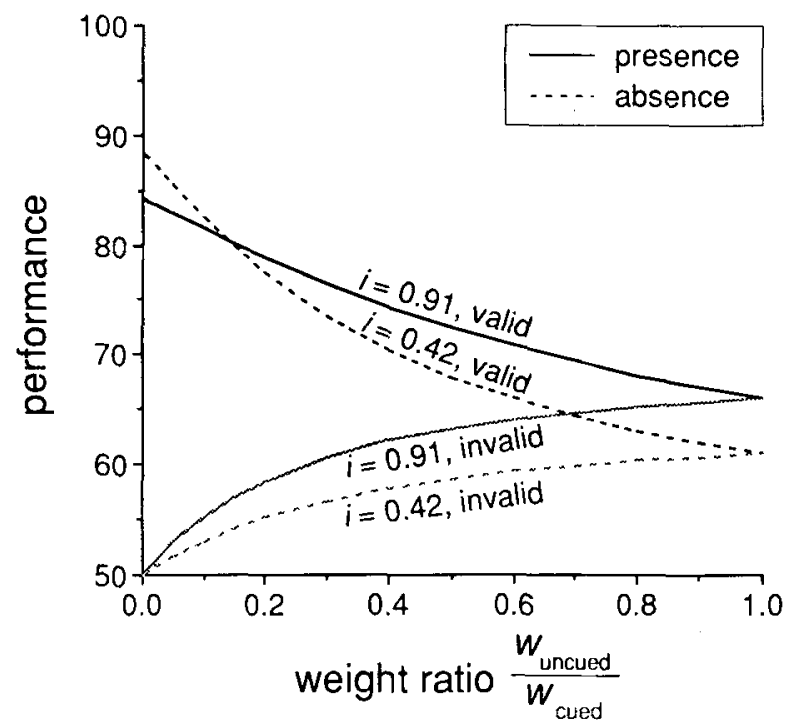

Figure A3. Predicted performance when selectivity for amplitude change is decreased. Model parameters are the same as in Table 5, except that $b=0.5$ instead of 0.1 . plus a covariance term that depends on the correlation of activities between fingertips $\left(\rho_{a}\right)$.

The subject makes a response by assuming that the larger of two observed pooled activities corresponds to the larger of the two stimulus arrays. The probability that the response is correct can be computed from the statistic $Z$.

\section{Predictions}

The model's predictions depend on six parameters: the minimum relative weight of inputs to the pooled activity $w_{\min } / w_{\max }$, the response of feature detectors to steady-state vibration $b$, the

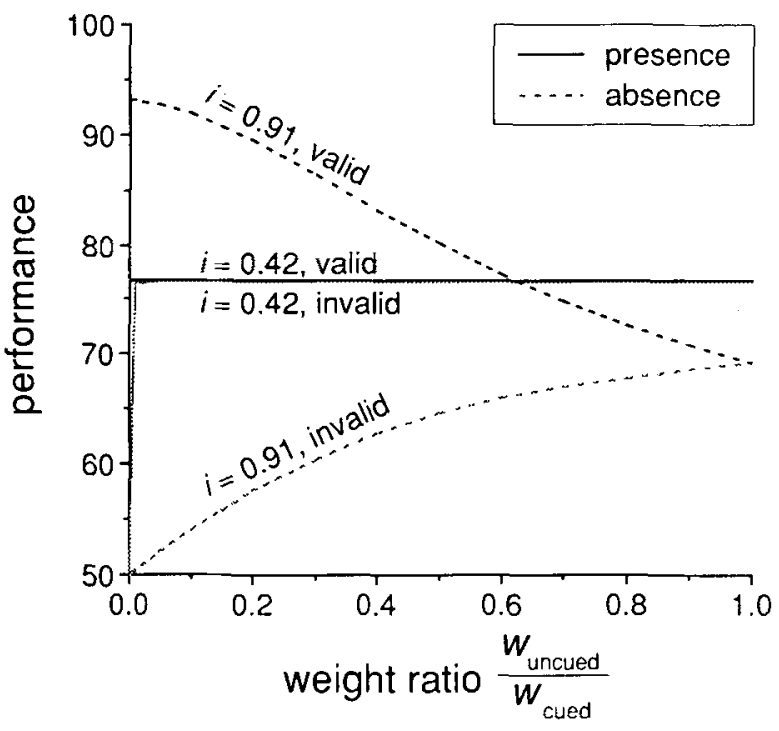

Figure A4. Predicted performance when correlation of activities representing different fingertips is decreased. Model parameters are the same as in Table 5, except that $\rho_{a}=0.1$ instead of 0.7 . 
correlation of activities between fingertips $\rho_{a}$, the ratio of mean to standard deviation of detector activities $\mu_{u} / \sigma_{u}$, and the parameters for the detector threshold distribution $\mu_{\theta}$ and $\sigma_{\theta}^{2}$.

In agreement with the observed attentional asymmetry, the model predicts that the relative weighting of cued and uncued fingertips affects the absence task much more than it affects the presence task. In Figure A2, the leftmost points on each graph represent the probability of a correct response when only the cued fingertip contributes to the pooled activity (selective attention). The rightmost points represent the model's predictions when all fingertips are given equal weight (divided attention). Overall performance on the presence task is nearly independent of weighting, whereas performance on the absence task is highest when the relative weight of the uncued fingertips is minimized. The basis of this asymmetry can be explained in terms of the behavior of the $Z$ statistic.

Combining the equations in Figure A1, and assuming that the feature detectors are highly selective,

$$
\mu_{a}(b+\mathrm{i})>\mu_{a}(b),
$$

the $Z$ statistic for the presence task on validly cued trials is approximated by

$$
\begin{aligned}
Z_{\text {presence, valid }} & \approx \frac{w_{\text {cued }} \mu_{a}(b+i)}{w_{\text {cued }} \sigma_{a}(b+i)} \\
& =\frac{\mu_{a}(b+i)}{\sigma_{a}(b+i)} .
\end{aligned}
$$

The cancellation of $w_{\text {cued }}$ in the numerator and denominator indicates that discriminability is independent of the relative weight-or attention-allocated to each fingertip. The corresponding approximation for invalidly cued trials reduces to the same expression.

This approximation points out a key difference in behavior between $Z$ and the Weber fraction. The "background" in $Z$, represented by the denominator variance, takes account of both stimulus arrays. In the presence task, the variance of the more intense array dominates, since there are no amplitude changes in the less intense array and the response to feature-absent stimuli is small. The Weber fraction, in contrast, represents only the less intense stimulus array in its denominator, so it is extremely sensitive to exactly how small the response is to steady-state vibration.

The corresponding approximations of the $Z$ statistics for the absence task are

$Z_{\text {absence, valid }}$

$\approx \frac{w_{\text {cued }} \mu_{a}(b+i)}{\sqrt{\left(w_{\text {cued }}^{2}+6 w_{\text {uncued }}^{2}\right)+\rho_{a}\left(6 w_{\text {cued }} w_{\text {uncued }}+12 w_{\text {uncued }}^{2}\right)}} \frac{\overline{\sigma_{a}(b+i)}}{\sigma^{2}}$

on validly cued trials, and

$Z_{\text {absence, invalid }}$
$\approx \frac{w_{\text {uncued }} \mu_{a}(b+i)}{\sqrt{\left(2 w_{\text {cued }}^{2}+5 w_{\text {uncued }}^{2}+\rho_{a}\left(10 w_{\text {cued }} w_{\text {uncued }}+8 w_{\text {uncued }}^{2}\right)\right.}} \frac{\sigma_{a}(b+i)}{}$

on invalidly cued trials. Decreasing the relative weight of the uncued fingertips cuts out most of the background variance; consequently, performance on validly cued trials is predicted to increase. Performance on invalidly cued trials is predicted to decrease, since the weight of the target fingertip is reduced. This is represented in the numerator of $Z_{\text {absence, invalid. }}$

Figure A3 shows that the degree of asymmetry predicted by the model depends on the feature detectors' selectivity for amplitude change. As $b$ is increased such that activity evoked by steady-state vibration $\mu_{a}(b)$ is no longer negligible (Equation 1), cuing is predicted to have a progressively greater effect on the presence task.

Figure A4, compared with Figure A2, shows the effect of the between-fingers correlation $\rho_{a}$ on the overall difficulties of the presence and absence tasks. As predicted by Equations 2, 3, and 4 , more positive values of $\rho_{a}$ result in lower performance on both validly and invalidly cued trials of the absence task, but do not affect performance on the presence task as long as $b$ is sufficiently small. The ratio of mean to standard deviation of detector activity $\mu_{u} / \sigma_{u}$ affects performance on the two tasks equally (Equations 2, 3, and 4).

(Manuscript received June 27, 1990; revision accepted for publication March 6, 1991.) 